\title{
CARACTERÍSTICAS MORFOMÉTRICAS, RENDIMENTO DE FILÉ E COMPOSIÇÃO QUIIMICA DA TRAÍRA
}

\author{
Karine Coelho de Araújo'; Luis Gabriel Alves Cirne ${ }^{2}$; Wisdeyvi Silva de Souza ${ }^{3}$; Jeruza Rodrigues da \\ Silva ${ }^{4}$; Rodrigo de Barros Feltran ${ }^{5}$; Denise Ribeiro de Melo ${ }^{6}$; Paulo Roberto Ribeiro de Melo ${ }^{7}$; \\ Edymeiko de Souza Maciel ${ }^{8}$.

\footnotetext{
1 Universidade Federal de Roraima, Boa vista, Roraima, Brasil, krine.xd@hotmail.com 2 Universidade Federal do Oeste do Pará, Santarém, Pará, Brasil, Lgabrielcirne@hotmail.com

3 Universidade Federal de Roraima, Boa vista, Roraima, Brasil, wisdeyviss@gmail.com ${ }^{4}$ Universidade Federal de Roraima, Boa vista, Roraima, Brasil, jeruzarodrigues.rr@hotmail.com ${ }^{5}$ Universidade Federal de Roraima, Boa vista, Roraima, Brasil, rodrigo.feltran@ufrr.br ${ }^{6}$ Universidade Federal de Roraima, Boa vista, Roraima, Brasil, demelo.ufr@@yahoo.com.br

7 Universidade Federal de Roraima, Boa vista, Roraima, Brasil, paulo.rocha@ufrr.br

8Universidade Federal de Roraima, Boa vista, Roraima, Brasil, edymeiko@gmail.com
}

RESUMO: Objetivou-se com este trabalho avaliar as características morfométricas, rendimento de filé e composição química da traíra (Hoplias malabaricus) com diferentes pesos. Foram utilizadas dezesseis traíras, em que os tratamentos $(T)$ foram em função do peso vivo dos animais constituídos por T1: traíra entre 0,400 e 0,550 kg e T2: traíra entre 0,600 e 0,800 kg, distribuídos em um delineamento inteiramente casualizado. $O$ comprimento total e padrão aumentaram $(P<0,05)$ à medida que se elevou o peso de abate. Houve correlação entre o peso de abate e o comprimento total e padrão com o peso de filé. Os animais com maiores pesos apresentaram $(P<0,05)$ menor teor de gordura, enquanto o teor de umidade, proteína e cinzas não foram afetados $(P>0,05)$. $O$ peso de abate e o comprimento total e padrão da traíra se correlacionam com o peso de filé e a carne da traíra é considerada magra e proteica e pode atender uma dieta saudável e nutritiva.

PALAVRAS-CHAVE: Composição centesimal, Correlação, Pescado.

\section{MORPHOMETRIC CHARACTERISTICS, FILLET YIELD AND CHEMICAL COMPOSITION OF TRAÍRA}

\begin{abstract}
The objective of this study was to evaluate the morphometric characteristics, fillet yield and chemical composition of traira (Hoplias malabaricus) with different weights. Sixteen traíras were used, in which treatments (T) were due to the live weight of the animals made of T1: traíra between 0.400 and $0.550 \mathrm{~kg}$ and T2: traíra between 0.600 and $0.800 \mathrm{~kg}$, distributed in a completely randomized design. The total and the standard length increased $(P<0.05)$ as the slaughter weight increased. There was a correlation between the slaughter weight and the total and the standard length
\end{abstract}


with the fillet weight. The animals with higher weights $(P<0.05)$ had lower fat content, while the moisture, protein and ash contents were not affected $(P>0.05)$. The traíra slaughter weight, total and the standard length are correlated with the fillet weight and the meat of traíra is considered lean and high-protein and can serve a healthy and nutritious diet.

KEYWORDS: Centesimal composition, Correlation, Fish.

\section{CARACTERÍSTICAS MORFOMÉTRICAS, RENDIMIENTO DEL FILETE Y COMPOSICIÓN QUÍMICA DE LA TARARIRA}

RESUMEN: El objetivo de este estudio fue evaluar las características morfométricas, rendimiento del filete y composición química de la tararira (Hoplias malabaricus) con diferentes pesos. Se utilizaron dieciséis tarariras, las cuales pasaron por tratamientos (T) en función del peso vivo de los animales constituidos por T1: tararira entre 0,400 y $0,550 \mathrm{~kg}$ y T2: tararira entre 0,600 y $0,800 \mathrm{~kg}$, distribuidos en un delineamiento completamente casualizado. La largura total y el patrón aumentaron $(P<0,05)$ a medida que se elevó el peso de sacrificio. Hubo correlación entre el peso de sacrificio, la largura total y el patrón con el peso del filete. Los animales con pesos mayores presentaron $(P<0,05)$ contenido menor de grasa, mientras que el contenido de humedad, proteína y cenizas no se afectaron $(P>0,05)$. El peso de sacrificio, la largura total y el patrón de la tararira se correlacionan al peso del filete y la carne de tararira se considera magra y proteica y puede atender una dieta sana y nutritiva.

PALABRAS CLAVE: Composición centesimal, Correlación, Pescado.

\section{INTRODUÇÃO}

As regiões brasileiras vêm se

especializando na criação de

determinados pescados, e novas

espécies nativas começam a fazer parte

da piscicultura nacional, onde o Brasil

possui entorno de 40 espécies de água

doce com capacidade para o cultivo.
Baldisserotto e Carvalho (2013) relatam que a maioria das espécies criadas no Brasil é de origem exótica, atribuindo isso a escassez de informações sobre o cultivo de espécies, o que de fato se torna surpreendente, pois o Brasil possui três mil espécies de peixes. A produção de espécies nativas no Brasil fica abaixo 
de $20 \%$, ao passo que, o maior produtor mundial de peixes, a Ásia, apresenta 95\% da produção composta por espécies nativas daquele (CARLSON, 2014).

Na década de 1980, a Companhia de Desenvolvimento dos Vales do São Francisco e do Parnaíba (CODEVASF) importou um pacote tecnológico de reprodução de peixes e o adaptou, tornando possível a reprodução artificial em cativeiros e, assim, a produção de alevinos de diversas espécies brasileiras. Além disso, o Brasil apresenta potencial para produção de peixes devido ao favorecimento de diversas características ambientais como volume de água disponível, clima favorável e grande biodiversidade (FARIA et al., 2013).

A traíra Hoplias malabaricus $(\mathrm{BLOCH}$, 1794) pertence à família Erythinidae, e sua presença é comum nas águas da América do Sul. É considerada nativa do Brasil, encontrada principalmente nos Estados do Amazonas, Tocantins, Mato Grosso, Pará, Rondônia e Roraima, onde habita tanto ambientes lóticos, como rios e cachoeiras, como ambientes estritamente lênticos, como lagos e reservatórios. Essa espécie possui o habito alimentar piscívoro; normalmente possuem o corpo roliço, com extremidades mais afiladas; ausência de nadadeira adiposa; comprimento padrão em torno de 275 mm, podendo atingir tamanho máximo de $80 \mathrm{~cm}$ de comprimento e $5 \mathrm{~kg}$ de peso vivo; resistente a baixos níveis de oxigênio na água e com ocorrência em lagos (SILVANO et al., 2001; OYAKAWA; MATTOX, 2009).

A baixa produção de espécies nativas também está relacionada ao baixo consumo devido à presença de espinhas intramusculares, dispondo de mais trabalho para a sua remoção, entretanto, no Estado de Minas Gerais, a traíra é considerada um dos pratos mais típicos da região, desmistificando o perigo do consumo de peixes com a presença de espinhos, que podem ser facilmente removidos por pessoas treinadas (KUBITZA et al., 2007). Diante desse contexto, o presente trabalho teve como objetivo avaliar as características morfométricas, rendimento de carcaça e 
composição química da traíra com diferentes pesos.

\section{MATERIAL E MÉTODOS}

O trabalho foi realizado no Laboratório de Tecnologia de Produtos de Origem Animal (TPOA) do Centro de Ciências Agrárias (CCA) da Universidade Federal de Roraima (UFRR), em Boa Vista, RR. Para a pesquisa foram utilizadas dezesseis traíras Hoplias malabaricus (BLOCH, 1794), em que os tratamentos $(T)$ foram em função do peso vivo dos animais constituídos por T1: traíra entre 0,400 e 0,550 kg e T2: traíra entre 0,600 e 0,800 kg.

Os peixes foram pescados na época seca, durante o dia, em um lago localizado nas dependências do campus Cauamé da própria instituição, no dia 24 de fevereiro de 2015, e, posteriormente, os peixes foram eutanasiados pela perfuração craniana utilizando um instrumento perfurante pontiagudo e, logo após, submetidos à hipotermia em caixa isotérmica com água e gelo até a sua morte. Para a captura dos exemplares de traíra se utilizou linha e anzol. Após essa etapa, os peixes foram acondicionados em outras caixas isotérmicas com gelo do tipo escama, em uma proporção peso e gelo de 1:2, e transportados até o Laboratório de TPOA.

No laboratório de TPOA os peixes foram pesados em balança eletrônica, para obtenção do peso total e, em seguida, foram realizadas as medidas biométricas dos animais, como comprimento total e padrão, e a relação entre estas características foram mensuradas de acordo com a metodologia preconizada por Lundstedt et al. (1997) e Hurlbut e Clay (1998), em que tais medidas foram obtidas com auxílio de um ictiômetro (graduado em cm) e um paquímetro (graduado em mm). A razão entre essas medidas foi calculada conforme Costa (2011), da seguinte forma: $\mathrm{CP} / \mathrm{CT}=$ comprimento padrão/comprimento total. Após a realização das medidas biométricas, cada exemplar foi individualmente abatido, da seguinte maneira: decapitado, eviscerado, retirado às nadadeiras, a cauda, as escamas, a pele 
e os ossos; e, em seguida, determinados o peso e o rendimento de filé (carcaça sem cabeça, sem pele, sem nadadeiras dorsais, ventrais e peitorais).

O processo de filetagem foi realizado por uma única pessoa previamente treinada, que consistiu da retirada da pele, realizada com o auxílio de um alicate com ponta fina e, posteriormente, a obtenção dos filés das duas laterais do peixe no sentido longitudinal, ao longo de toda a extensão da coluna vertebral, realizada com o auxílio de uma faca (MACEDO-VIEGAS et al., 1997). As amostras de filés foram individualmente identificadas, embaladas em sacos plásticos e armazenadas a $-80{ }^{\circ} \mathrm{C}$ em ultrafreezer até o início das análises. No preparo das amostras para análises, os filés foram descongelados dentro dos sacos plásticos em incubadora B.O.D. a $10{ }^{\circ} \mathrm{C}$ por 12 horas, e, dos mesmos, amostras foram retiradas para determinação da composição centesimal.

Para determinação da composição centesimal, amostras do filé foram liofilizadas por 72 horas e após a liofilização, moídas para a obtenção da amostra laboratorial. A composição centesimal da carne foi determinada em umidade, proteína, gordura (extrato etéreo) e minerais, segundo metodologia preconizada pela AOAC (1995).

O delineamento experimental utilizado foi inteiramente casualizado com 2 tratamentos e 8 repetições, totalizando 16 unidades experimentais. Os resultados foram avaliados por meio de análises de variância e teste de Tukey, sendo a significância das médias obtida pelo teste $\mathrm{F}$ até $5 \%$ de probabilidade, utilizando-se o programa estatístico Sisvar (FERREIRA, 2011). Entretanto, para as correlações, os resultados foram avaliados por meio do teste de Pearson, a $1 \%$ de probabilidade, utilizando o programa estatístico sigma plot 11 (SIGMAPLOT, 2008).

\section{RESULTADOS E DISCUSSÃO}

Houve influência $(P<0,01)$ do peso ao abate no comprimento total (CT) e padrão (CP) (Tabela 1). 
O valor médio de comprimento total, de 36,87 cm, está na faixa registrada por Santos et al. (2001), que ao trabalharem com traíra Hoplias malabaricus (BLOCH, 1794) encontraram valores entre 25 e $53 \mathrm{~cm}$; por Acunã et al. (2011), que ao pesquisarem sobre traíras na Venezuela, encontraram valores entre 30 e $45 \mathrm{~cm}$ para animais com peso entre 500 e 1500 g, respectivamente; e por Loureiro e Hahn (1996), que ao analisarem sobre dieta e atividade alimentar de traíra no Paraná, observaram valores entre 27 a $45 \mathrm{~cm}$ para peixes adultos.

Carvalho et al. (2002) ao estudarem sobre alimentação de traíras no Pantanal Sul Mato-Grossense, encontraram valores entre 13,5 e 26,0 cm para comprimento padrão em peixes pesando de 50 a 390 g. Oliveros e Rossi (1991) consideraram peixes adultos com comprimento total acima de $23,0 \mathrm{~cm}$ ao pesquisarem sobre traíra na Argentina, o que demonstra que os peixes desta pesquisa, possivelmente, eram adultos.

De acordo com Maghelly et al. (2014), é de fundamental importância o estudo do formato do corpo, características morfométricas e rendimento corporal para a caracterizar as espécies. No entanto, Diodatti (2008) relata que as medidas morfométricas, o peso de abate, os diferentes métodos de processamento e o conhecimento sobre rendimento do peixe são importantes para a indústria de processamento e para produtor, além disso, o estudo sobre as características de carcaça é um meio para avaliar o potencial produtivo das propriedades e pode ser utilizada de forma indireta para caracterização da carcaça, sem abater o animal.

Tabela 1. Características morfométricas de traíra com diferentes pesos.

\begin{tabular}{|c|c|c|c|c|}
\hline \multirow{2}{*}{ Item } & \multicolumn{2}{|c|}{ Peso (g) } & \multirow{2}{*}{$\mathrm{P}^{1}$} & \multirow{2}{*}{$C V(\%)^{2}$} \\
\hline & $400-550 \mathrm{~g}$ & $600-800 \mathrm{~g}$ & & \\
\hline CT & $34,50 a$ & $39,25 b$ & 0,000 & 3,87 \\
\hline$C P$ & $28,50 a$ & $33,50 b$ & 0,003 & 9,21 \\
\hline $\mathrm{CP} / \mathrm{CT}$ & 0,826 & 0,852 & 0,365 & 6,43 \\
\hline
\end{tabular}

Médias seguidas de mesma letra na linha não diferente estatisticamente entre si $(P<0,05)$ pelo teste de Tukey a $5 \%$ de probabilidade.

${ }^{1} \mathrm{P}=$ probabilidade; ${ }^{2} \mathrm{CV}=$ coeficiente de variação. 
Existe alta correlação $(P<0,01)$ entre o peso de abate com comprimento total e padrão e com o peso de filé (Tabela 2), ou seja, à medida que o peso de abate aumenta, maior é o comprimento total e padrão e, consequentemente, o peso de filé.

Tabela 2. Correlações entre o peso de abate (PAB), comprimento total (CT), comprimento padrão $(C P)$, relação $C P / C T$, peso do filé (PFILE) e rendimento de filé (RFILE) em percentual das traíras estudadas.

\begin{tabular}{lccccc}
\hline Variável & CT & CP & CP/CT & PFILE & RFILE \\
& & & & & \\
\hline PAB $(\mathrm{g})$ & $0,97^{\star \star}$ & $0,98^{\star \star}$ & $-0,20$ & $0,96^{\star \star}$ & $-0,11$ \\
CT $(\mathrm{cm})$ & - & $0,98^{\star \star}$ & $-0,32$ & $0,93^{\star \star}$ & $-0,16$ \\
CP $(\mathrm{cm})$ & & - & $-0,13$ & $0,93^{\star \star}$ & $-0,14$ \\
CP/CT & & & - & $-0,18$ & 0,13 \\
PFILE (g) & & & & - & 0,15 \\
\hline
\end{tabular}

**significativo a 1\% pela Correlação de Pearson.

Santos et al. (2001) ao estudarem sobre a composição química e rendimento de filé de traíra Hoplias malabaricus $(\mathrm{BLOCH}$, 1794), encontraram resultados semelhantes aos deste trabalho, em que a correlação entre o peso e comprimento total foi alta e satisfatória em relação ao peso do filé, porém, esses autores verificaram correlação existente entre o peso, comprimento total e rendimento de filé para estimar o peso do filé de traíra.

A relação peso-comprimento é uma ferramenta importante, que permite avaliar o crescimento dos peixes (SILVA
JÚNIOR et al., 2007). De acordo com Lizama e Ambrósio (1999) é de fundamental importância o conhecimento sobre a relação peso-comprimento, quando estão associadas há outros elementos como fator de condição, recrutamento, crescimento e mortalidade, pois este conhecimento disponibiliza informações necessárias para o estudo da biologia dos peixes, seu manejo racional e preservação de seu ambiente.

Não houve influência $(P>0,01)$ do peso ao abate no rendimento de filé de traíra (Tabela 3) com diferentes pesos. 
Tabela 3. Peso de abate e peso e rendimento de filé de traíra com diferentes pesos.

\begin{tabular}{lccrc}
\hline \multirow{2}{*}{ tem } & \multicolumn{2}{c}{ Peso $(\mathrm{g})$} & \multirow{2}{*}{$\mathrm{P}^{1}$} & \multirow{2}{*}{$\mathrm{CV}(\%)^{2}$} \\
\cline { 2 - 3 } & $400-550 \mathrm{~g}$ & $600-800 \mathrm{~g}$ & & \\
\hline Peso ao abate (g) & $466,87 \mathrm{a}$ & $677,50 \mathrm{~b}$ & 0,000 & 9,81 \\
Peso de filé (g) & $161,00 \mathrm{a}$ & $234,99 \mathrm{~b}$ & 0,000 & 7,96 \\
Rendimento de filé (\%) & 34,62 & 34,76 & 0,890 & 5,71 \\
\hline
\end{tabular}

Médias seguidas de mesma letra na linha não diferente estatisticamente entre si $(P<0,05)$ pelo teste de Tukey a $5 \%$ de probabilidade.

${ }^{1} \mathrm{P}=$ probabilidade; ${ }^{2} \mathrm{CV}=$ coeficiente de variação.

Rendimento de filé superior ao encontrado neste trabalho foi observado por Santos et al. (2001), que avaliaram o rendimento de filé de traíra Hoplias malabaricus (BLOCH, 1794), obtendo média de 44,33\%, entretanto, os machos apresentaram valores superiores 48,63\%, em relação as fêmeas que obtiveram 46,12\%, demonstrando diferença entre os sexos. A influência do sexo em relação ao rendimento de filé foi descrita também por Reidel et al. (2010) ao trabalhar com rendimento corporal de jundiás alimentados com diferentes níveis de proteína e energia, criados em tanques redes.

Contudo, outros estudos, embora com espécies diferentes, mostram rendimentos semelhantes como o encontrado por Silva et al. (2009), entre 33 e 36\%, em tilápia-doNilo (Oreochromis niloticus); por Carneiro et al. (2004), entre 29 a 35\%, em Jundiá (Rhamdia quelen); e por Fernandes et al. (2010), entre 31 e 32\%, em tambaqui (Colossoma macropomum). Clement e Lovell (1994) relatam valores encontrados na literatura variando de 25,4 a 42\%, sendo que o rendimento de filé encontrado nesta pesquisa, de 34,69\%, está na faixa supracitada.

Os valores médios da composição centesimal do filé de traíra obtidos com diferentes pesos apresentou alteração $(P<0,05)$ apenas no teor de gordura (Tabela 4).

Com base nestes dados, o filé de traíra Hoplias malabaricus pode ser considerado peixe proteico, devido aos altos valores de proteína, e como peixe magro, em virtude dos baixos teores de gordura, ocupando a categoria D (> 20\% de proteína e < $5 \%$ de gordura) na classificação citada por Stanby 
(1962), que incluiu o teor de proteína para categorizar os pescados.

Santos et al. (2001) ao estudarem a composição química de traíra Hoplias malabaricus observaram valor inferior para gordura, de apenas 0,84\%. Este mesmo autor cita a importância da classificação do peixe pelo teor de gordura, pois dependendo da quantidade esta pode influenciar positivamente ou negativamente a aceitação pelo mercado consumidor e o desempenho produtivo, podendo também alterar a palatabilidade da carne do peixe.

Sales e Sales (1990) analisaram a composição química de dez espécies de água doce de interesse comercial nos açudes do nordeste brasileiro, sendo as espécies estudadas tilápia (Tilapia melanopleura), o tucunaré comum (Cichla ocellaris), a sardinha (Triportheus angulatus), $\quad$ curimatã comum (Prochilodus cearenses), a pescada do piauí (Plagioscion squamosissimus), a traíra (Hoplias malabaricus), o piau comum (Leporinus frideric), o piau verdadeiro (Leporinus sp.), a cangati (Trachycorystes sp.) e a branquinha (Curimatã sp.). Em relação ao ponto de vista nutricional, as dez espécies apresentaram alto teor de proteína, porém, a traíra Hoplias malabaricus ficou em segundo lugar entre as dez estudadas com percentual de 21,5\% de proteína, evidenciando que esta espécie possui ótimo valor nutricional, entretanto, em relação à gordura, a traíra apresentou média de 2,5\%, sendo classificada como peixe magro, com pouco valor calórico.

Tabela 4. Composição centesimal do filé de traíra com diferentes pesos.

\begin{tabular}{|c|c|c|c|c|}
\hline \multirow{2}{*}{ Item } & \multicolumn{2}{|c|}{ Peso $(\mathrm{g})$} & \multirow{2}{*}{$P^{1}$} & \multirow{2}{*}{$C V(\%)^{2}$} \\
\hline & $400-550 g$ & $600-800 \mathrm{~g}$ & & \\
\hline Umidade & 75,08 & 75,46 & 0,631 & 1,95 \\
\hline Proteína & 19,74 & 20,23 & 0,456 & 5,90 \\
\hline Gordura & $2,00 a$ & $1,46 b$ & 0,044 & 25,77 \\
\hline Cinzas & 1,77 & 2,04 & 0,258 & 22,69 \\
\hline
\end{tabular}

Médias seguidas de mesma letra na linha não diferente estatisticamente entre si $(P<0,05)$ pelo teste de Tukey a $5 \%$ de probabilidade.

${ }^{1} \mathrm{P}=$ probabilidade; ${ }^{2} \mathrm{CV}=$ coeficiente de variação. 
O teor de cinzas encontrados no filé da traíra foi de $1,77 \%$ para os peixes que apresentaram peso entre 400 a $500 \mathrm{~g} \mathrm{e} 2,04 \%$ para os com pesos entre 600 a 800 g. Contreras-Guzmán (1994) cita que os peixes de água doce apresentam variações de 0,90 a 3,39\% de frações de cinzas em sua composição centesimal. O valor de cinzas encontrados neste trabalho foi compatível com os valores supracitados. Com relação aos minerais, Simão et al. (2007) relatam que a carne de pescado é uma excelente fonte de cálcio e fósforo, além de apresentar outros minerais em pequenas quantidades como potássio, sódio, manganês, zinco, cobre, cobalto, iodo e ferro.

\section{CONCLUSÃO}

O peso de abate e o comprimento total e padrão da traíra se correlacionam com o peso de filé e a carne da traíra é considerada magra e proteica e pode atender uma dieta saudável e nutritiva.

\section{REFERÊNCIAS}

ACUÑA, J.; GONZÁLEZ, E. J.; ÁLVARES, M. A.; PEÑAHERRERA, C.; BARRETO, M. Evaluácion del contenido de aminoácidos em guabina (Hoplias malabaricus), Bloch 1784, capturadas em el embase Hipereutrófico de Suata (Estado Aragua, Venezuela). Revista Científica, v.21, p.265-274, 2011.

ASSOCIATION OF OFFICIAL ANALYTICAL CHEMISTS - AOAC. Official methods of analysis, 16.ed. Washington: DC, 1995. 1011 p.

BALDISSEROTTO, B.; CARVALHO, L. Espécies nativas para piscicultura no Brasil. Santa Maria: Editora UFSM, 2013. 608 p.

CARLSON, V. Anuário Brasileiro da Pesca e Aquicultura. Rio de Janeiro: ACEB, 2014. 136 p.

CARNEIRO, P. C. F.; MIKOS, J. D.; BENDHACK, F.; IGNÁCIO S. A. Processamento do jundiá Rhamdia quelem: rendimento de carcaça. Revista acadêmica: Ciências Agrárias e Ambientais, v.5, p.11-17, 2004.

CARVALHO, L. N.; FERNANDES, C. H. V.; MOREIRA, V. S. Alimentação de Hoplias malabaricus (Bloch, 1794) (Osteichthyes, Erythrinidae) no rio Vermelho, Pantanal Sul MatoGrossense. Revista Brasileira de Zoociências, v.4, p.227-236, 2002.

CLEMENT, S.; LOVELL, R. T. Comparison of culture Nile tilapia (Oreochromis niloticus) and channel 
catfish (Ictalurus punctatus). Aquaculture, v.119, p.299-310, 1994.

CONTRERAS-GUZMÁN, E. S. Bioquímica de pescados e derivados. Jaboticabal: FUNEP, 1994. 409 p.

COSTA, A. C. Medidas morfométricas na avaliação de pesos e rendimentos corporais de Pacu Piaractus mesopotamicus e Tambaqui Colossoma macropomum. Lavras, 2011. 64 f. Dissertação (Mestrado em Zootecnia) - Universidade Federal de Lavras, Lavras, 2011.

FARIA, R. H. S.; MORAIS, M.; SORANNA, M. R. G. S.; SALLUM, W. B. Manual de criação de peixes em viveiros. Brasília: Codevasf, 2013. 136 p.

FERNANDES. T. R. C.; DORIA, C. R. C.; MENEZES, J. T. B. Características de carcaça e parâmetros de desempenho do tambaqui (Colossoma macropomum, Cuvier, 1818) em diferentes tempos de cultivo e alimentado com rações comerciais. Boletim do Instituto de Pesca, v.36, p.45-52, 2004.

FERREIRA, D.F. Sisvar: A computer statistical analysis system. Ciência e Agrotecnologia, v.35, p.1039-1042, 2011.

HURLBUT, T.; CLAY, D. Morphometric and meristic differences between shallow and deep-water populations of white hake (Urophycis tenuis) in the southern Gulf of St. Lawrence. Canadian Journal of Animal Science, v.55, p.2274-2282, 1998.
KUBITZA, F; ONO, E. A; CAMPOS, J. L. Os caminhos da produção de peixes nativos no Brasil: Uma análise da produção e obstáculos da piscicultura. Panorama da Aquicultura, v.17, p. 1423, 2007.

LIZAMA, A. P.; AMBRÓsio, A. M. Relação peso-comprimento e estrutura da população de nove espécies de characidae na planície de inundação do Alto Rio Paraná, Brasil. Revista Brasileira de Zoologia, v.16, p.779-788, 1999.

LOUREIRO, V. E.; HAHN, N. S. Dieta e atividade alimentar da traíra, Hoplias malabaricus (Bloch, 1794) (Osteichthyes, Erythrinidae), nos primeiros anos de formação do reservatório de Segredo - PR. Acta Limnologica Brasiliensia, v.8, p.195-205, 1996.

LUNDSTEDT, L. M.; LEONHARDT, J. H.; DIAS, A. L. Alterações morfométricas induzidas pela reversão sexual em tilápias do Nilo Oreochromis niloticus. Revista Unimar, v.19, p.461-472, 1997.

MACEDO-VIEGAS, E. M.; SOUZA, M. L. R.; KRONKA, S. N. Estudo da carcaça de tilápia do Nilo (Oreochonis niloticus), em quatro categorias de peso. Revista Unimar, v.19, p.863-870, 1997.

OLIVEROS, O. B.; ROSSI, L. M. Ecologia trófica de Hoplias malabaricus malabaricus (Pisces, Erythrinidae). Revista de Ciências Naturales del Litoral, v.22, p.55-68, 1991. 
OYAKAWA, O. T:; MATTOX, G. M. Revision of the Neotropical trahiras of the Hoplias lacerdae species-group (Ostarioophysi: Characiforme: Eryyhrinidae) with descriptions of two new species. Neotropical Ichthyology, v.7, p.117-140, 2009.

RECHSTEINER, M. O.; MAIA, H. G.; EVOY, Z. F.; SCHOFFEN, E. D. B. Características morfométricas e rendimento corporal do suruvi Steindachneridion scriptumagrupados por sexo. Boletim do Instituto de Pesca, v.40, p.419-430, 2014.

REIDEL, A.; ROMAGOSA, E.; FEIDEN, A.; BOSCOLO, W, R.; COLDEBELLA, A.; SIGNOR, A. A. Rendimento corporal e composição química de jundías alimentados com diferentes níveis de proteína e energia na dieta, criados em tanques-rede. Revista Brasileira de Zootecnia, v.39, p.233-240, 2010.

SALES, R. O.; SALES, A. M. Estudos da composição química e rendimento de dez espécies de pescado de água doce de interesse comercial nos açudes do nordeste brasileiro. Revista Ciência Agronômica, v.21, p.27-30, 1990.

SANTOS, A. B.; MELO, J. F. B.; LOPES, P. R. S.; MALGARIM, M. B. Composição química e rendimento do filé da traíra (Hoplias malabaricus). Revista FZVA, v.7/8, p.140-150, 2001.

SIGMAPLOT. For windows, version 11.0. San Jose: Systat Software, 2008.
SILVA JÚNIOR, M. G.; CASTRO, A. C. L.; SOARES, L. S.; FRANÇA, V. L. Relação peso-comprimento de espécies de peixe do estuário do Rio Paciência da ilha do Maranhão, Brasil. Boletim laboratório de Hidrologia, v.20, p.3138, 2007.

SILVA, F. V.; SARMENTO, N. L. A. F.; VIEIRA, J. S.; TESSITORE, A. J. A.; OLIVEIRA, L. L. S.; SARAIVA, E. P. Características morfometricas, rendimentos de carcaça, filé, vísceras e resíduos em tilápia-do-nilo em diferentes faixas de peso. Revista Brasileira de Zootecnia, v.38, p.14071412, 2009.

SILVANO, R. A. M.; OYAKAWA, O. T.; AMARAL, B. D.; BEGOSSI, A. A. Peixes do Alto Rio Juruá (Amazonas, Brasil). São Paulo: Editora da Universidade de São Paulo: Impressa Oficial do Estado, 2001. 304 p.

SIMÕES, M. R.; RIBEIRO, C. F. A.; RIBEIRO, S. C. A.; PARK, K. J.; MURR, F. E. X. Composição físico-química, microbiológica e rendimento do filé de tilápia tailandesa (Oreochromis niloticus). Ciência e Tecnologia de Alimentos, v.27, p.608-613, 2007.

STANBY, M. R. Proximate composition of fish. In: Heen, E.; Kreuzer, R. (Org.) Fish in nutrition. London: Fishing News Books Ltd., 1962, 59 p. 